\title{
Cognitive networks: a new access scheme which introduces a Darwinian approach
}

\author{
Philippe Jacquet \\ Alcatel-Lucent Bell Labs \\ Paul Muhlethaler \\ Philippe.Jacquet@alcatel-lucent.comPaul.Muhlethaler@inria.fr
}

\begin{abstract}
In this paper we present a new approach for cognitive radio. In the usual approach the secondary network is in charge of monitoring the channel to determine whether or not the primary network is active in the area. If it is not, the secondary network is allowed to use the spectrum. In the new access scheme we propose, the primary network encompasses the techniques which allow it to capture the bandwidth even if the secondary network is transmitting in the area. The access scheme of the primary network preempts the secondary network activity. We present an access scheme which preempts the IEEE 802.11 decentralized scheme. This protocol is a generalized Carrier Sense Multiple Access scheme using active signaling. Instead of only sensing the carrier, this algorithm also transmits bursts of signal which may be sensed by the other nodes. If so, they give up the selection process. We show that this scheme preempts the IEEE 802.11 decentralized access scheme if the bursts transmitted by the node in the primary network are made up of special sequences which alternate between bursts of signal and periods of sensing. These sequences called $(d, k)$ sequences [1] encompass a minimum number $d$ and a maximum number of $k$ successive zeros during which the node senses the channel to find other possible concurrent transmissions. In practice we use $d=0$ and $k$ depends on the duration of the IEEE 802.11 interframe space and the duration of a signaling burst. We compute the number of $(0, k)$ sequences with respect to the length $n$ of the sequence. We also show that $(d, k)$ sequences (with $2 d>k$ ) can be used if, by mistake, during the signaling phase one burst is not detected. We evaluate the number of such sequences.

Index Terms-Cognitive network, primary and secondary network, Carrier Multiple Access (CSMA), active signaling, preemptive access, $d(d, k)$ sequences.
\end{abstract}

\section{INTRODUCTION}

Economically, the existence of cognitive networks is justified by the fact that many spectra are not fully used by their dedicated users, and therefore allowing secondary users access will give the opportunity to fully use the bandwiths and provide more spectrum to users. This is particularly true when part of the bandwidth is reserved for applications that have not yet been developed. The time necessary for such applications to come onto market may be long or may simply never occur (due to reasons other than technological ones), and precious bandwidth may simply be wasted for a substantially long period. Therefore the FCC has edicted that any bandwidth dedicated to a new usage should also accept secondary users in the context of cognitive networks.

Cognitive networks are radio networks where each band of frequency is occupied by two groups of users: the pri- mary users that form the primary network and the secondary users that form the secondary network. The primary users are supposed to have priority over the secondary users: i.e. the performance of the primary network should be protected against the traffic of the secondary network. By protection we mean that the performance of the primary network should be guaranteed independently of the demand from the secondary network. Furthermore, the throughput and occupancy of the secondary network should vanish when the traffic load of the primary network increases. In other words the secondary users are only allowed to take the blank periods left by the primary users.

The problem is that the protocol used by the primary users, in short the primary protocol, often comes after a standardization process that ignores the secondary users. The consequence is that the design of the secondary protocol is sometimes harder and more costly than the design of the primary protocol because the secondary protocol must indeed embed the features of the primary protocol in order to be able to give priority to primary users. This is a kind of "AntiDarwinian" approach because the technological burden is on the secondary users while the market success is guaranteed to the primary users. In an analogy with evolution, it is as if the most sophisticated species should automatically lose against the less sophisticated. The result would be that the spectrum may not be fully used leading to economic failure.

In this paper we present an access scheme which offers an alternative and more natural approach to cognitive networks which we call the "Darwinian Approach". The approach consists in identifying an already standardized protocol for the secondary user, for example the IEEE 802.11 standards, and then designing a primary protocol that naturally preempts the secondary protocol. Pre-empting the IEEE 802.11 standard [2] is not difficult as it only requires defining a primary protocol with a smaller interframe space than the IEEE 802.11 Distributed Interframe Spacing (DIFS). With this strategy the advantages are:

1) the market succes of the secondary user is guaranteed, since the secondary protocol is already available; therefore the spectrum is immediately fully used;

2) the technological investment for the primary users is guaranteed by its enforced priority over the secondary users.

In contrast to previous approaches [3] we adopt a strategy 
where the sharing rules are mostly implemented in the primary network. Usually the secondary network encompasses mechanisms which allow this network to resume its transmission if the primary network is not using the channel. Here the secondary network uses the IEEE 802.11 decentralized MAC scheme. The key is a preemptive access implemented in the primary network. When both networks coexist the secondary network captures the bandwidth when, in a given area, there is no activity in the primary network (or even no network nodes at all). The secondary network can use the bandwidth without any modification to its normal mode of operation.

The paper is organized as follows. Section II presents related work. Section III describes the technique used by the primary network to share the spectrum between its nodes and to preempt the bandwidth from the secondary users. This MAC access scheme is inspired by the access scheme of the HiPERLAN type 1 standard [4] and this technique is governed by binary sequences. We derive the conditions under which the primary network preempts the channel. We show that these conditions imply that the binary sequences be special sequences that we call $(d, k)$ sequences. In section IV we compute the number of $(d, k)$ sequences. We focus on $(0, k)$ sequences. We also study $(d, k)$ sequences with $2 d>k$ because with these sequences the access scheme can mis-detect one burst. Section V concludes the paper.

\section{RELATED WORK}

In cognitive radio we can distinguish two different issues. The first issue is sensing, which is a key feature in cognitive radio networks since it allows the secondary network to be aware of the existence of primary nodes within a given area. The other issue is medium access, which is also a central issue since smart access techniques can be used by cognitive radio networks to share the medium efficiently.

Traditional approaches limit the transmitter power of the secondary nodes so that the transmitted power remains below a given threshold at a given distance from the transmitter. However with the mobility of nodes, it is difficult to constrain transmitter power as new nodes may appear at any time. To cope with this issue, the FCC tried to introduce a new metric: the interference temperature. The goal was to keep the interference experienced by the receivers below a given threshold. The secondary nodes must ensure that their transmission plus the already existing noise does not exceed the interference temperature limit at the primary nodes. This can be controlled for instance by a sensor network [3] which monitors the energy in different locations of the network. However, it has been argued that this concept of temperature tends to increase the interference in the frequency bands where it would be used and that it is not a workable concept. Thus in 2007 the FCC gave up trying to build coexistence rules based on interference temperature. Energy detection is the most common type of spectrum sensing. The presence or the absence of transmissions by the primary network must be established based on measurements of the radio signal. We are faced with the usual paradigm of detection which must be done with the contradictory goals of minimizing the probability of missing a detection while also minimizing the probability of a false alarm (detecting a primary node when no such node exists). The detection usually uses the inherent periodicities such as the modulation rate, carrier frequency, etc. see [5], [6]. If the secondary nodes have access to information about the signal sent by the primary network, then the matched filter is the optimal detection method, see [7]. There are many other sensing techniques [3], [8], [9], [10]

As regards cognitive access techniques, an interesting classification distinguishes between DAB (Direct Access Based) protocols and DSA (Dynamic Spectrum Allocation) protocols. In $\mathrm{DAB}$ protocols there is no global network optimization, each sender-receiver pair in the cognitive network tries to take the best advantage of the available resources using very simple algorithms. In contrast, DSA protocols look for the optimization of a global criterion and use much more complex schemes.

In DAB protocols we can again distinguish between contention-based protocols and coordination-based protocols. In contention-based protocols the cognitive nodes exchange their sensing results using a simple handshake [11], [12], [13]. Then the sender-receiver pair analyzes available resources and negotiates the channel that will be used to communicate. In coordination-based protocols each cognitive node shares its channel usage information with its neighbors to gain a better view of the overall utilization of the channels, [14], [15], [16], [17].

DSA protocols exploit complex optimization algorithms to optimize a global criterion. For instance DSA protocols may use graph coloring theory [18], game theory [19], stochastic theory [20] and genetic algorithms.

\section{NETWORK DESCRIPTION OF PRIMARY-SECONDARY PROTOCOLS}

\section{A. Secondary network based on the IEEE 802.11 standard}

The IEEE 802.11 decentralized medium access scheme uses a linear backoff. At the end of a transmission i.e. after the end of the packet (followed by its acknowledgement packet for point-to-point packets), a node which has pending packets waits for the interframe spacing. After this time interval, the node waits for a random number of collision slots (it decrements its backoff time) and after this time interval elapses, the node sends a packet ${ }^{1}$. If another node sends a packet before the first node has finished decrementing its backoff, the first node resumes decrementing its backoff after the current transmission. We have an example of this situation in Figure 1. Station B has a backoff of 4 mini-slots whereas station $\mathrm{C}$ has a backoff of 6 mini-slots. Thus station $\mathrm{B}$ sends

\footnotetext{
${ }^{1}$ The IEEE 802.11 is actually linear in the collision window even if the binary exponential back-off doubles the collision window when there is a collision
} 
its packet at the end of station A's transmission (including the acknowledgement for point-to-point packets) and 4 minislots. Station C waits for the end of station B's transmission and waits for an additional 2 mini-slots before sending its packet. We notice that the interval between a packet and its acknowledgement packet is a Short InterFrame Space (SIFS). After the acknowledgement packet, the other nodes which are waiting to transmit a packet must wait for a Distributed InterFrame Space (DIFS) to start decrementing their backoff in the collision window $(\mathrm{CW})$. This mechanism requires that the duration of an SIFS is smaller than the duration of a DIFS, with this situation the backoff is not decremented between a packet and its acknowledgement.

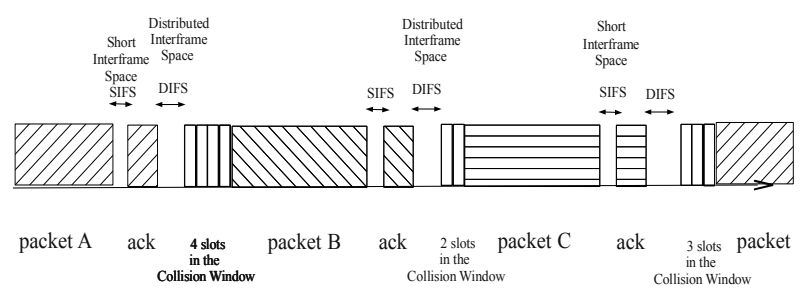

Fig. 1. Example of the linear back-off used by the IEEE 802.11 MAC.

\section{B. Primary network CSMA technique with active signaling}

The generalized CSMA technique we propose introduces the concept of active signaling, which is the original basis of HiPERLAN type 1 [4]. Rather than performing the carrier sensing during a random time interval, the technique we propose allows the protocol to switch between bursts of signal and periods of sensing. Since a node switches between listening and transmitting, a half-duplex transceiver is sufficient. The fundamental rule of CSMA remains the same: as soon as an energy above a given threshold is sensed then the station quits the selection process and waits for the end of the current selection process with the transmission of a packet to start competing again. The active signaling technique adds bursts to improve the selection process of CSMA; when CSMA needs an overhead $0(n)$ to select among $n$ contenders it can be shown [21] that the active signaling technique only needs $0(\log (n))$.

A simple way to describe the station's activity during the signaling period is to code the signaling period with " 1 " or " 0 ". 1 represents a transmission burst and " 0 " a sensing interval. Thus the signaling burst " 101 " is composed of a signaling burst, a sensing interval and another signaling burst. The active signaling burst which is shown in Figure 2 can be represented by the binary sequence " 111001110000110 ". We can notice that this sensing rule is such that the station with the highest binary sequence is selected for transmission. Then comes the second highest and so on.

The interframe space between a packet and its acknowledgement is still a SIFS but the time interval between the

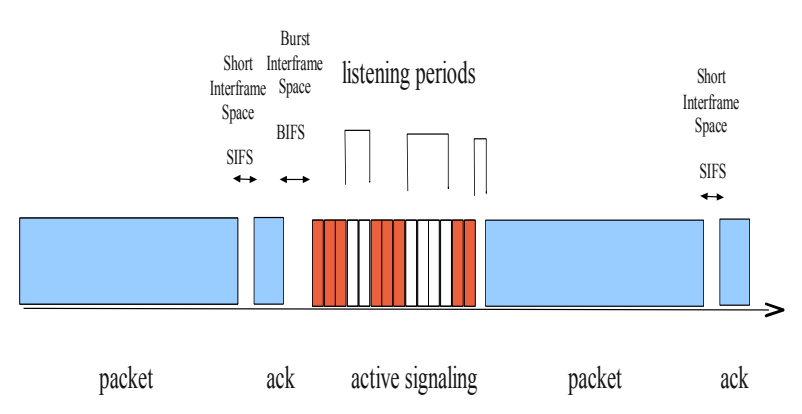

Fig. 2. Signaling burst in the generalized CSMA technique proposed.

end of the acknowledgement packet and the beginning of the signaling part is a Burst InterFrame Space (BIFS). We assume that every node always uses the same binary sequence to govern its access and we call this sequence the node access sequence. Of course, the nodes with larger access sequences would have more access opportunities than nodes with smaller access sequences. To cope with this fairness issue we can add the following rule: after its transmission, a node must observe an idle interval of at least one Large Burst InterFrame Space (LBIFS) to be allowed to start its signaling phase again (see Figure 3). Thus a node in the primary network can only have a single packet in the "train" of packets. This succession of signaling bursts, packets and acknowledgements separated by Burst InterFrame Spaces (BIFSs) is called an "epoch". Two successive epochs are separated by a Long Burst InterFrame Space (LBIFS). If we assume that a primary user always uses the same sequence to access the channel, then the order in which the primary users send their packet is the same in any epoch. In other words if node A transmits before node B in one epoch this will always the same in every epoch. The proof of this simple property is left to the reader.

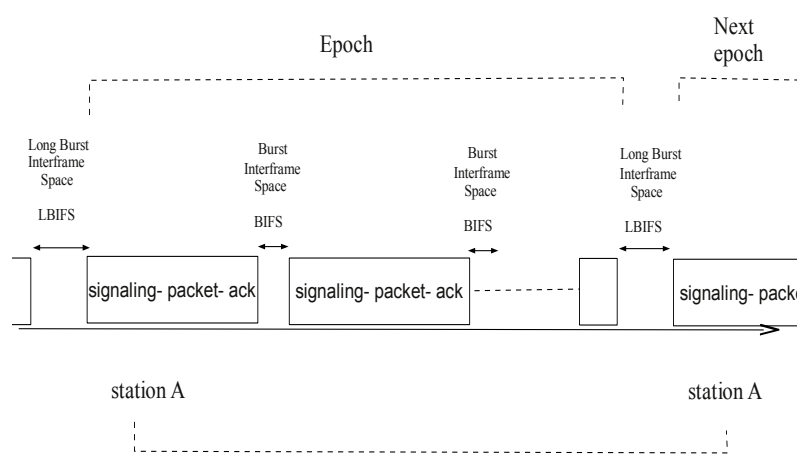

Fig. 3. Fairness with the signaling burst CSMA technique proposed. A station can only transmit a single packet in one "epoch"

In order to preserve the priority of the primary network 
over the secondary network, the duration of the LBIFS must be smaller than the duration of the DIFS. The duration of the LBIFS should also be larger than the duration of BIFS. This implies that nodes even with a smaller access sequence than the access sequence used by node A will have opportunities to send their packets. This mechanism creates "epochs", two successive epochs being separated by an idle period with a duration of at least one BIFS. Another solution to obtain fair access is to draw the binary sequences used in the primary network at random.

In the following we study the timing constraints to ensure that the secondary network nodes only get access to the channel when the primary network nodes are not transmitting. The first constraint is that the BIFS is smaller than the DIFS. This gives a prioritized access to the primary network nodes. Moreover, to ensure that a BIFS can not be interpreted as a DIFS, it is mandatory that the first bit of the binary sequences used in the generalized CSMA be a '1'. A slightly less strict condition is that the duration of the BIFS plus the duration of the beginning of listening bursts at the head of the binary sequence have a duration smaller than the duration of the DIFS.

If we satisfy these constraints, the generalized CSMA technique used for the primary network will preempt the access of a secondary user using the decentralized IEEE 802.11 MAC protocol. However another constraint is necessary to ensure that the secondary network can not insert transmissions in the signaling period of the primary network. To avoid that, the binary sequence used in the primary network must not contain too many successive zeros. The duration of a sequence of listening coded by successive zeros should be shorter than a DIFS, see Figure 1. In this case the nodes using the IEEE 802.11 access scheme do not start decrementing their backoff during the sensing intervals of the signaling bursts of the primary network and no packet of the secondary network can be inserted.

In Table I we present the values used in the IEEE 802.11 for the SIFS and DIFS. We also propose values for the duration of the BIFS $(30 \mu s)$ and the LBIFS $(40 \mu s)$.

\begin{tabular}{|l|c|}
\hline Burst or interframe & duration \\
\hline 1 burst & $9 \mu \mathrm{s}$ \\
\hline SIFS & $10 \mu \mathrm{s}$ \\
\hline BIFS & $30 \mu \mathrm{s}$ \\
\hline LBIFS & $40 \mu \mathrm{s}$ \\
\hline DIFS & $50 \mu \mathrm{s}$ \\
\hline
\end{tabular}

TABLE I

DURATION OF THE BURSTS AND THE INTERFRAMES

An interesting tool to govern access in the primary network is $(d, k)$ binary sequences [1]. These binary sequences contain strings of ' 0 ' where 0 appears at least $d$ times and at most $k$ times between two successive ' 1 '. For our application we use $d=0$ and the maximum value of $k$ can be easily computed with the duration of the Distributed Interframe Space of the IEEE 802.11 MAC scheme $d_{D I F S}$ and the duration of the signaling burst $d_{s b}, k=k_{1}=\left\lfloor\frac{d_{D I F S}}{d_{s b}}\right\rfloor$. If the primary network uses the CSMA technique with active signaling and $\left(0, k_{1}\right)$ binary sequence then we are sure that during the signaling period of a primary network node there is no listening period (i.e. idle period) of length greater than $d_{D I F S}$. In other words, the IEEE 802.11 access scheme can not start decrementing its backoff in the signaling period of the primary network and thus no transmission can be inserted in the active signaling period of the primary network.

In Figure 4 we show the successive transmissions on the channel when primary and secondary users share the channel. In the begining of the time interval shown in this figure, the primary users have no packet to send. Thus the secondary users can send their packets at the beginning of this time intervall. We notice that these packets (and their acknowledgements) are separated by DIFSs. When a primary user becomes active it sends its packet after a LBIFS and then this user has priority on the channel over the secondary users. The other primary users follow this transmission using BIFSs and thus creating an "epoch". Since the BIFSs and the listening periods in the active signaling part of the primary users are smaller than the DIFSs, no secondary users can access the channel in the epoch. This epoch finishes by a DIFS if no primary user still has packets to send ${ }^{2}$ or by a LBIFS if primary users still have packets to send. The entire succession of interframe spaces is shown in Figure 4. The transmission unit for the secondary users is a fraction of a collision window, the packet itself and its acknowledgement repesented in Figure 4 by CW-PA. The transmission unit for the primary users is the signaling bursts, the packet itself and its acknowledgement, repesented in Figure 4 by S-PA.

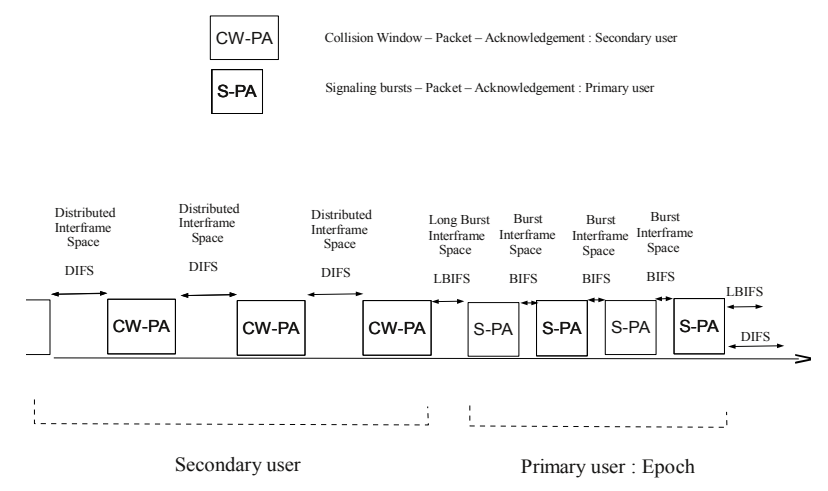

Fig. 4. Time interval when secondary and primary users share the channel. At the beginning of this interval only secondary users have pending packets.

\footnotetext{
${ }^{2}$ The same node can only send one packet during an epoch for fairness reasons
} 


\section{IV. $(d, k)$ SEQUENCES ANALYSIS}

In this section we compute the theoretical minimal length of the $(d, k)$ sequence needed to encode $N$ distinct access sequences, in order to guarantee collision free access for a network of size $N$. In fact we adopt the reverse approach we compute as $X_{n}$ the maximum number of distinct $(d, k)$ sequences of length $n$, and then we find $n$ such that $X_{n} \geq N$. First we compute for the $(0, k)$ sequences, and then we generalize for the $(d, k)$ sequences that can be applied in noisy conditions.

\section{A. $(0, k)$ sequences}

In this section, we recall previously obtained results [1] concerning $(0, k)$ sequences; for simplicity we use the same notations. We compute the number of $(0, k)$ sequences for a given length $n$. In these strings the number of zeros between two consecutive 'ones' is smaller than or equal to $k$. We denote $W_{n}$ as the number of $(0, k)$ sequences of length $n$ which start by a one. Let $l$ be an integer, we also denote $X_{n}$ the number of $(0, k)$ sequences of length $n$ which start by up to $l$ zeros.

We introduce

$$
A_{k}(z)=\sum_{n=0}^{k} z^{n}=\frac{z^{k+1}-1}{z-1},
$$

and let us denote $\rho_{0}$ the real non-negative root of $z A_{k}(z)-1$, we set $\lambda_{0}=\frac{1}{\rho_{0}}$. From [1] we have the following estimation

$$
\begin{aligned}
W_{n} & =\frac{\lambda_{0}^{n+1}}{\lambda_{0}+A_{k}^{\prime}\left(\rho_{0}\right) \rho_{0}}+0\left(r^{n}\right) \\
X_{n} & =\frac{A_{l}\left(\rho_{0}\right) \lambda_{0}^{n+1}}{\lambda_{0}+A_{k}^{\prime}\left(\rho_{0}\right) \rho_{0}}+0\left(r^{n}\right) .
\end{aligned}
$$

for some $r<\lambda_{0}$.

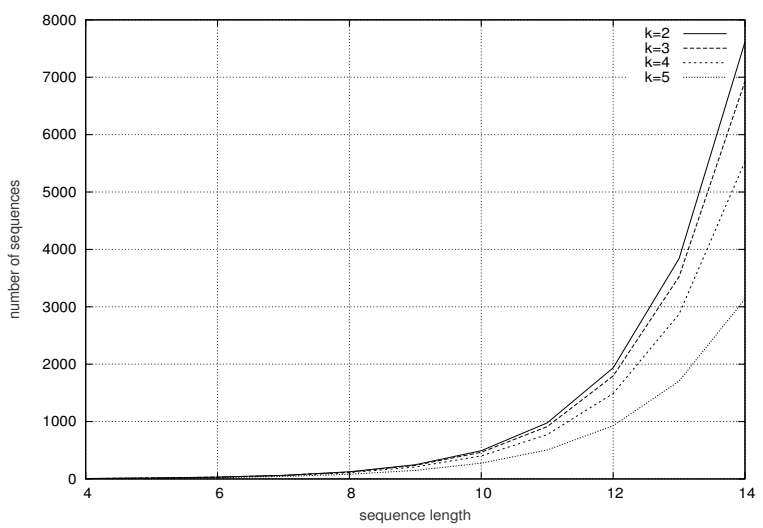

Fig. 5. Number of $(0, k)$ sequences of length $n$.

In Figure 5 we give the number of $(0, k)$ sequences of length $n$ starting with one 'one' for $k=2,3,4,5$. In this Figure we can observe the reduction in the number of sequences when $k$ decreases. If we wish to have more sequences we can allow up to $l$ zeros at the beginning of the $(0, k)$ sequences.
In Figure 6, we compare the number of $(0, k)$ sequences of length $n$ starting with one 'one' with the number of $(0, k)$ sequences of length $n$ which start with up to $l$ zeros. With the values given in Table I we take $k=5$ and $l=2$. We observe that setting the constraint of starting the $(0, k)$ sequence with one 'one' whereas the constraint concerning the maximum duration of a silent interval $\left(<d_{D I F S}\right)$ allows one to use up to $l=2$ zeros at the head of the sequence which results in significantly more sequences. This can be useful if the primary network contains a large number of nodes.

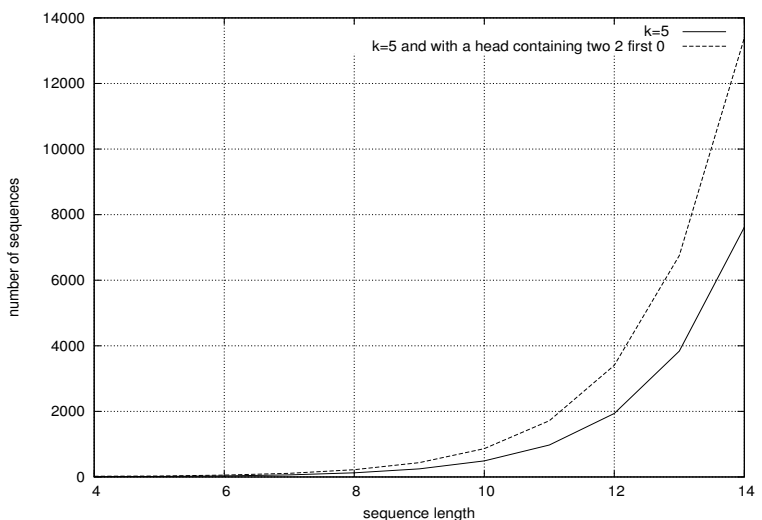

Fig. 6. Comparison between the number of $(0,5)$ sequences of length $n$ with 1 at the head and with the number of $(0,5)$ sequences of length $n$ with up to 2 zeros at the head followed by a 1 .

\section{B. $(d, k)$ sequences}

If by mistake during the signaling phase, one burst is not detected by a node which is in a listening period this may lead to two nodes sending a packet simultaneously (collision). This can be avoided by using $(d, k)$ sequences. It is easy to show that two $(d, k)$ sequences have a hamming distance greater or equal to 2 if and only if $2 d>k$. With this condition the mis-detection of one burst can be tolerated without creating a collision. We can thus use sequences composed as follows: one 'one' followed by between $d$ and $k$ zeros (this pattern can be iterated an undefinite number of times). The sequence finishes with a zero. We denote by $Y_{n}$ the number of such sequences of length $n$ starting by a 'one' and $Z_{n}$ the number of $(d, k)$ sequences of length $n$ starting by up to $l$ zeros. Using the methodology previously employed [1] and denoting $C_{d, k}(z)=$ $\sum_{i=d}^{i=k} z^{n}=\frac{z^{k+1}-z^{d}}{z-1}$ it can be shown that

$$
\begin{gathered}
Y_{n}=\frac{1}{\mu_{0}+C^{\prime}\left(\tau_{0}\right) \tau_{0}}+0\left(r^{n}\right) . \\
Z_{n}=\frac{A_{l}\left(\tau_{0}\right)\left(1+\tau_{0}\right) \mu_{0}^{n+1}}{\mu_{0}+C_{d, k}^{\prime}\left(\tau_{0}\right) \tau_{0}}+0\left(r^{n}\right) .
\end{gathered}
$$

with $0<\tau_{0}<1$ satisfying $\tau_{0} C_{d, k}\left(\tau_{0}\right)=1$ and $\mu_{0}=1 / \tau_{0}$ and some $r<\mu_{0}$.

In Figure 7 we show the number of $(3,5)$ sequences of length $n$ starting with one 'one' and the number of $(3,5)$ sequences with up to 2 zeros at the head. We observe that the 


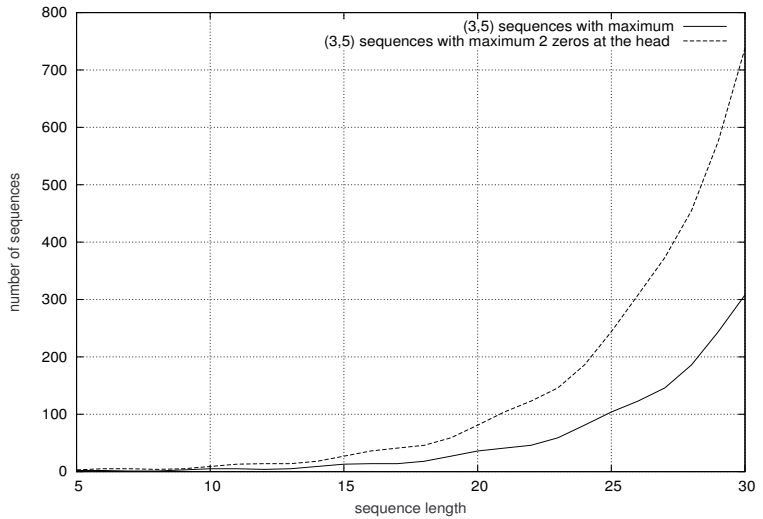

Fig. 7. Number of $(3,5)$ sequences of length $n$ starting with one 'one' and number of $(3,5)$ sequences with up to 2 zeros at the head.

number of available sequences is very significantly reduced compared to the number of $(0,5)$ sequences as shown in Figure 6. If the primary network contains a large number of nodes it may be better to cope with collisions than to use $(3,5)$ sequences because in this case we must set $n$ to a large value. Thus we have to pay a large signaling overhead for each transmission.

\section{CONCLUSION}

In this paper we have proposed a new scheme for cognitive radio in which the complexity is implemented in the primary network. The active signaling technique is used by the primary network to preempt the channel when the secondary network uses it. The access is fair among the nodes of the primary network. The nodes of the primary network preempt the bandwidth from the nodes of the secondary network. We study the timing constraints to obtain this property. We also define the rule to obtain a fair distribution among the nodes in the primary network if these nodes always use the same pattern in the signaling phase.

The main constraint to ensure that the primary nodes always have priority when they access the channel is that there is no idle interval in the signaling phase of the primary network greater than the Distributed InterFrame Space (DIFS). The primary network must use $(d, k)$ binary sequences in which two successive ones are separated by at least $d$ zeros and at most $k$ zeros to build the signaling burst. Here we must use $d=0$ and $k=\left\lfloor\frac{d_{D I F S}}{d_{S b}}\right\rfloor$. We study the number of these sequences depending on their length $n$. We investigate how the constraints can be slightly relaxed and the benefit in terms of the number of additional sequences which can be used. We also consider the case where a primary node may miss the detection of one burst (in exactly one interval). To cope with such a case, we propose using $(d, k)$ with $2 d>k$. For a given length, we compute the number of available sequences. We observe that the number of sequences is greatly reduced. Thus it may be better to allow a few collisions to occur and to resolve them by retransmitting rather than using $(d, k)$ sequences with $2 d>k$ which leads to a large overhead if a large number of sequences is needed.

\section{REFERENCES}

[1] P. Jacquet and B. Szpankowski, "On (d, k) sequences not containing a given word," in Proc. of IEEE ISIT, Seattle, 2006.

[2] "IEEE Standard for Information Technology-Telecommunications and Information Exchange Between Systems-Local and Metropolitan Area Networks-Specic Requirements Part 11: Wireless LAN Medium Access Control (MAC) and Physical Layer (PHY) Specications," IEEE Std. 802.11-2007 (Revision of IEEE Std. 802.11-1999).” December 2007.

[3] S. Haykin, "Cognitive radio: Brain-empowered wireless communications.” IEEE J. Sel. Areas Comm, vol. 23 no 2, pp. 201-220, 2005.

[4] "ETSI HIPERLAN functional specifications, ETS 300-654," June 1996.

[5] W. Gardner, "Signal interception: a unifying theoretical framework for feature detection," IEEE Transactions on Communications, vol. 36 no 8, pp. 897-906, 1988.

[6] M. Oner and F. Jondral, "Air interface recognition for a software radio system exploiting cyclostationarity," in PIMRC, vol. 3, Barcelona, 2004, pp. 1947-1951.

[7] J. Proakis, Digital Communications. New-York: McGraw-Hill, 2001.

[8] B. Farhang-Boroujeny, "Filter bank spectrum sensing for cognitive radios," IEEE Transactions on Signal Processing, vol. 56, no. 5, pp. $1801-1811$, may 2008.

[9] H. Li, C. Li, and H. Dai, "Quickest spectrum sensing in cognitive radio," in Information Sciences and Systems, 2008. CISS 2008. 42nd Annual Conference on, march 2008, pp. $203-208$.

[10] H. Kim and K. Shin, "Fast discovery of spectrum opportunities in cognitive radio networks," in DySPAN 2008. 3rd IEEE Symposium on New Frontiers in Dynamic Spectrum Access Networks, oct 2008, pp. 1 -12 .

[11] J. Jia, Q. Zhang, and X. Shen, "HC-MAC: A hardware-constrained cognitive MAC for efficient spectrum management," in IEEE Journal on Selected Areas in Communications, vol. 26, no. 1, jan 2008, pp. 106-117.

[12] X. Wang, P.-H. Ho, and A. Wong, "Towards efficient spectrum sensing for cognitive radio through knowledge-based reasoning," in New Frontiers in Dynamic Spectrum Access Networks, 2008. DySPAN 2008. 3rd IEEE Symposium on, 2008, pp. $1-8$.

[13] H. B. Salameh, M. Krunz, and O. Younis, "MAC protocol for opportunistic cognitive radio networks with soft guarantees." vol. 8, no. 10, oct 2009, pp. 1339-1352.

[14] H. Su and X. Zhang, "Cross-layer based opportunistic MAC protocols for QoS provisionings over cognitive radio wireless networks." in IEEE Journal on Selected Areas in Communications, vol. 26, no. 1, jan 2008, pp. $118-129$.

[15] C. Cordeiro and K. Challapali, "C-MAC: A cognitive MAC protocol for multi-channel wireless networks," in New Frontiers in Dynamic Spectrum Access Networks, 2007. DySPAN 2007. 2nd IEEE International Symposium on, 2007, pp. $147-157$.

[16] T. Chen, H. Zhang, G. Maggio, and I. Chlamtac, "Cogmesh: A clusterbased cognitive radio network," in New Frontiers in Dynamic Spectrum Access Networks, 2007. DySPAN 2007. 2nd IEEE International Symposium on, 2007, pp. $168-178$.

[17] J. Zhao, H. Zheng, and G.-H. Yang, "Distributed coordination in dynamic spectrum allocation networks," in New Frontiers in Dynamic Spectrum Access Networks, 2005. DySPAN 2005. 2005 First IEEE International Symposium on, 2005, pp. $259-268$.

[18] H. Zheng, "Collaboration and fairness in opportunistic spectrum access," in in Proc. of IEEE International Conference on Communications, 2005, pp. 3132-3136.

[19] C. Zou and C. Chigan, "On game theoretic DSA-driven MAC for cognitive radio networks," in Computer Communication Journal, vol. 32, no. 18, dec 2009, pp. 1944-1954.

[20] Q. Zhao, L. Tong, and A. Swami, "Decentralized cognitive mac for dynamic spectrum access," in First IEEE International Symposium on New Frontiers in Dynamic Spectrum Access Networks, DySPAN 2005, pp. 224-232.

[21] P. Jacquet, P. Minet, P. Muhlethaler, and R. N., "Priority and collision detection with active signaling: The Channel Access Mechanism of HIPERLAN," Wireless Personal Communications, vol. 4, pp. 11-25, 1997. 